
podem melhorar o Agronegócio do Brasil"

\title{
ANÁLISE DE FUNCIONAMENTO DO INTERCOOLER NO RENDIMENTO DE TORQUE E POTÊNCIA EM MOTORES TURBO DIESEL
}

\author{
Luana Ximenes Figueira $^{(1)}$ (Ixfigueira@hotmail.com) , Sebastião Luiz da Silva Viana ${ }^{(1)}$ \\ (sebastian.com@hotmail.com.br), André Luiz Vicente Carvalho ${ }^{(1)}$ (andrelvcarvalho@hotmail.com).
}

(1) Universidade Candido Mendes (UCAM); Engenharia Mecânica

RESUMO: Este trabalho apresenta a importância do intercooler, componente responsável pela melhoria do desempenho de motores diesel turbo alimentados. Seu objetivo principal é destacar a melhor performance e relevante ganho de torque e potência quando compactado o intercooler ao motor, através de testes realizados no dinamômetro inercial de rolo do laboratório da Universidade Candido Mendes da cidade de Campos dos Goytacazes. Seu funcionamento utiliza a permuta de calor e, consequentemente, resfriamento do ar que vem aquecido do compressor, antes de ser admitido na câmara de combustão do motor. Desta forma, o ar tornase mais denso para que, com isso, se tenha mais massa de ar no mesmo volume da câmara de combustão, $e$ consequentemente, uma melhor queima, aumentando o torque e a potência do motor. Com todo este processo de melhoria na combustão, os benefícios ficam evidentes, trazendo eficiência e performance em grande escala, representados por um aumento de aproximadamente $24,4 \%$ no torque e $32,1 \%$ na potência desenvolvida pelo motor testado.

PALAVRAS-CHAVE: INTERCOOLER, MOTOR DIESEL, TURBOCOMPRESSOR, TORQUE, POTÊNCIA.

\section{PERFORMANCE ANALYSIS OF INTERCOOLER IN TORQUE AND TURBO DIESEL ENGINE}

ABSTRACT: This paper presents the importance of intercooler, component responsible for improving the performance of turbocharged diesel engines. Its main objective is to highlight the best performance and relevant torque and power gain when compacting the engine intercooler, through tests performed in the inertial dynamometer of the laboratory of Candido Mendes University of Campos dos Goytacazes. Its operation utilizes heat exchange and, consequently, cooling of the heated air coming from the compressor before being admitted to the engine combustion chamber. In this way, the air becomes denser so that more air is massed in the same volume as the combustion chamber, and consequently a better burn, increasing the engine torque and horsepower. With all this combustion improvement process, the benefits are evident, bringing efficiency and performance on a large scale, represented by an increase of approximately $24.4 \%$ in torque and $32.1 \%$ in horsepower developed by the engine tested.

KEYWORDS: INTERCOOLER, DIESEL ENGINE, TURBOCOMPRESSOR, TORQUE, POWER. 


\section{XIX \\ CONEMI}

\section{INTRODUÇÃO}

Os motores de combustão por compressão, como é o caso dos motores diesel, não possuem frente de chama única. Nestes motores, a combustão ocorre de forma simultânea em vários pontos da câmara de combustão gerado pelo processo de injeção do diesel diretamente no cilindro, quando o pistão está no fim do processo de compressão do ar, previamente admitido. O combustível, no estado líquido é inserido de forma pulverizada a elevadas pressões por meio de injetores e atomizadores em pequenas gotas dentro da câmara de combustão; estas pequenas gotículas são vaporizadas e misturadas ao ar que, em virtude das condições termodinâmicas, pressão e temperatura, contidas no interior do cilindro, possibilita a autoignição da mistura.

O ar admitido nos cilindros, que compõe a mistura ar combustível e são responsáveis diretamente na qualidade da queima e combustão, é empurrado pelo turbo compressor, que faz parte do objeto de estudo desta pesquisa, levando maior quantidade de ar aos cilindros. O ar que sai do compressor para os cilindros alcança maiores temperaturas, já que a turbina se utiliza dos gases de exaustão para seu funcionamento, o aumento da pressão faz elevar temperatura, além de outras fontes de calor no compartimento do motor. Para tornar este ar mais frio antes da entrada nos cilindros e melhorar o processo de queima na combustão, que é a fonte geradora de força do motor, utiliza-se o intercooler, um trocador de calor capaz de diminuir a temperatura do ar, sem perda de pressão significativa tornando-o mais denso, ou seja, maior massa de ar em um mesmo volume.

Melhorar a queima com mais massa de ar no mesmo volume admitido nos cilindros, garante uma maior expansão dos gases na combustão, impondo mais força sobre os pistões, o que resulta em mais torque e mais potência do motor com a mesma quantidade de combustível consumida, ou seja, maior desempenho e rendimento.

Para descobrir a real diferença de eficiência do automóvel utilizando em condições originais e sem a utilização do intercooler, foram realizados testes no dinamômetro, do tipo rolo inercial, aonde foram escolhidos dois testes de cada condição. Após a realização dos testes, se faz a média dos resultados obtidos e são gerados gráficos para análise comparativa. 


\section{XIX \\ CENEMI}

\section{MATERIAIS E MÉTODOS}

\subsection{Materiais}

Para a realização dos testes no carro, utilizou-se:

- Veículo modelo Chevrolet S10 motor 2.8 turbo diesel, com intercooler, injeção eletrônica Common Rail, Ano 2017;

- Dinamômetro Inercial de Rolo Dynotech 720i.

\subsubsection{Veículo utilizado}

Para análise, foi escolhida uma caminhonete Chevrolet S10 motor 2.8 do ano 2017, apresentada na Figura 1, com motor diesel, equipada com turbocopressor, intercooler e injeção eletrônica Common Rail.

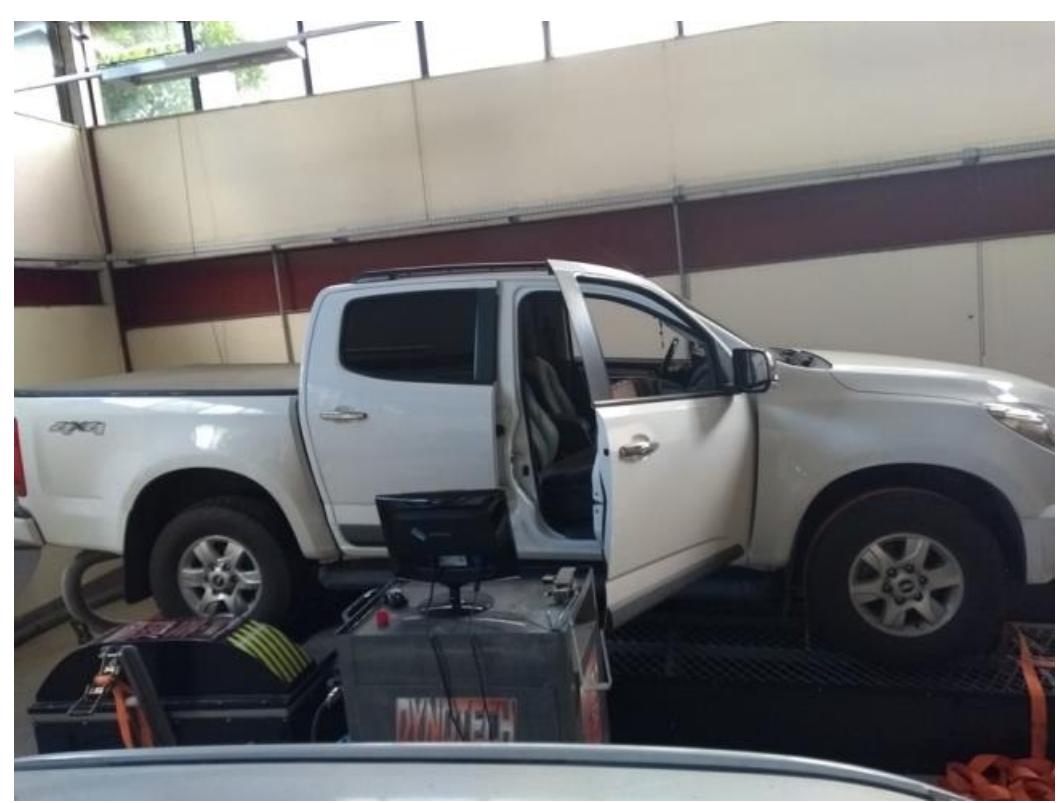

FIGURA 1. Caminhonete Chevrolet S10. Fonte: Autor (2018).

As especificações técnicas deste motor que foram utilizadas para os estudos se encontram na Tabela 1. 


\section{XIX \\ CENEMI}

TABELA 1. Quadro de Especificações do Motor.

\begin{tabular}{|c|c|}
\hline \multicolumn{2}{|c|}{ Especificações técnicas do Motor 2.8 CTDI GM S10 } \\
\hline Combustível & Diesel \\
Instalação & Dianteiro \\
Disposição & Longitudinal \\
Número de cilindros & 4 em linha \\
\hline Número de válvulas & 4 por cilindro \\
\hline Taxa de compressão & $16,5: 1$ \\
\hline Cilindrada & $2776 \mathrm{~cm}^{3}$ \\
\hline Comando de válvulas & Duplo no cabeçote, correia dentada \\
\hline Alimentação & Injeção direta \\
\hline Aspiração & Turbocompressor \\
\hline Diâmetro do cilindro & $94 \mathrm{~mm}$ \\
\hline Curso do pistão & $100 \mathrm{~mm}$ \\
\hline Potência do fabricante & $200 \mathrm{cv}$ a $3600 \mathrm{rpm}$ \\
\hline Torque do fabricante & $51 \mathrm{kgfm} \mathrm{a} 2000 \mathrm{rpm}$ \\
\hline Torque específico & $18,37 \mathrm{kgfm} / \mathrm{litro}$ \\
\hline Potência específica & $72,05 \mathrm{cv} / \mathrm{litro}$ \\
\hline Peso/potência & $10,21 \mathrm{~kg} / \mathrm{cv}$ \\
\hline Relação Diferencial & $40,04 \mathrm{~kg} / \mathrm{kgfm}$ \\
\hline Rotação máxima & $4400 \mathrm{rpm}$ \\
\hline Fonte: GM Chevrot &
\end{tabular}

Fonte: GM Chevrolet (2018).

\subsubsection{Dinamômetro Inicial de Rolo}

Para realização dos testes foi utilizado o dinamômetro, Dynotech 720i inercial de rolo, modelo ilustrado na Figura 2, disponível no Laboratório de Motores de Combustão Interna da Universidade Candido Mendes. 


\section{XIX \\ CONEMI}

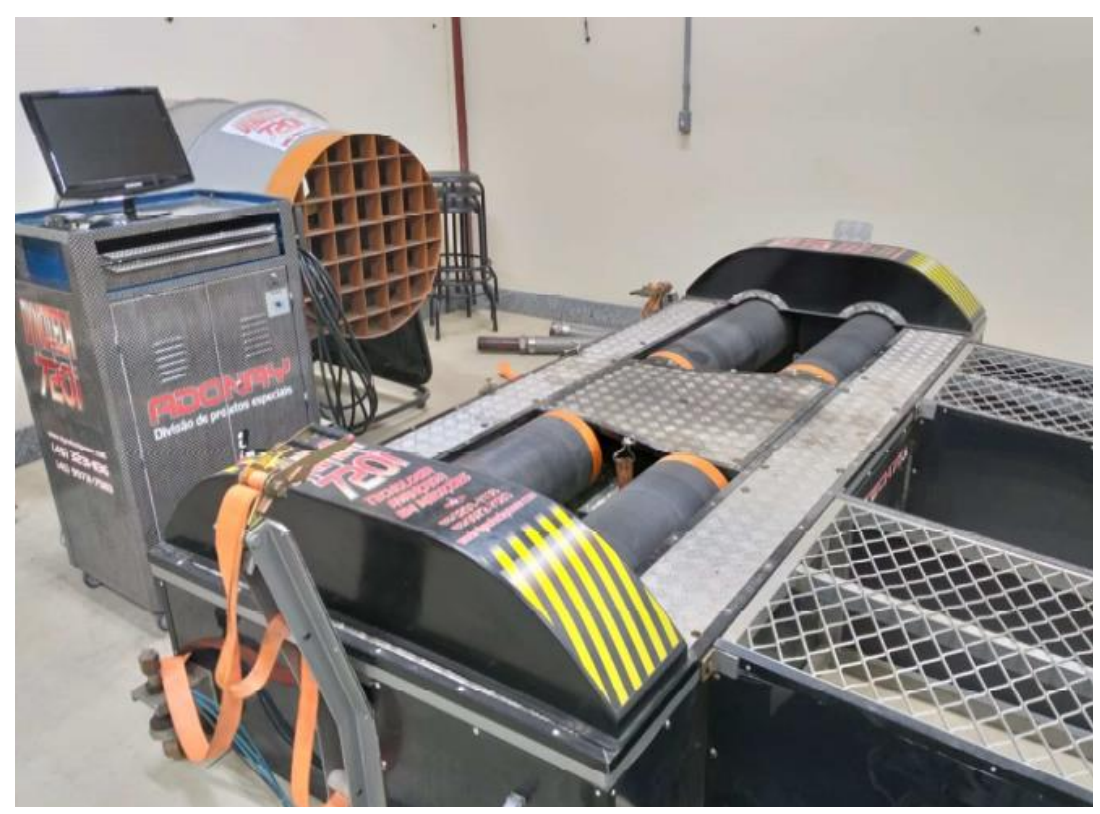

FIGURA 2. Dinamômetro Dynotech 720i inercial de rolo. Fonte: Autor (2018).

O veículo, através de uma rampa, é posicionado sobre uma plataforma e sobre os quatro cilindros de inércia do equipamento. Seu ventilador, posicionado em frente ao veículo, funciona progressivamente com variador de frequência automático ou manual, além de possuir hélices de ângulos variáveis, simulando o movimento do automóvel em circunstâncias reais, aumentando e diminuindo o fluxo de ar de acordo com a velocidade simulada. Os parâmetros; valores de torque e potência são coletados e demonstrados, em tempo real, através do software do equipamento. São geradas tabelas, as quais servem de referência para a geração de gráficos. Estes dados servem como bases de comparação dos testes realizados para apresentação dos resultados.

\subsection{Métodos}

Foram realizados testes com o motor diesel turbo intercooler 2.8, da caminhonete Chevrolet S10, onde o combustível utilizado foi o diesel S10. A primeira etapa consiste em submeter o motor a testes no dinamômetro para que o software forneça os dados necessários para estudos. Esses testes foram realizados utilizando o automóvel em condições normais e originais de fábrica. Na segunda etapa, os testes foram realizados sem o intercooler, ou seja, foi desconectada a mangueira de entrada de ar do intercooler, que vem da saída de ar do compressor e também foi desconectada a mangueira de saída de ar do intercooler, que entra para admissão nos cilindros. Para manter o fluxo de ar do compressor para admissão nos cilindros, sem passar pelo intercooler, foram instaladas mangueiras, conexões e abraçadeiras, conforme as Figuras 3 e 4. 


\section{$\mathbf{X I X}$ \\ CIONEMI}

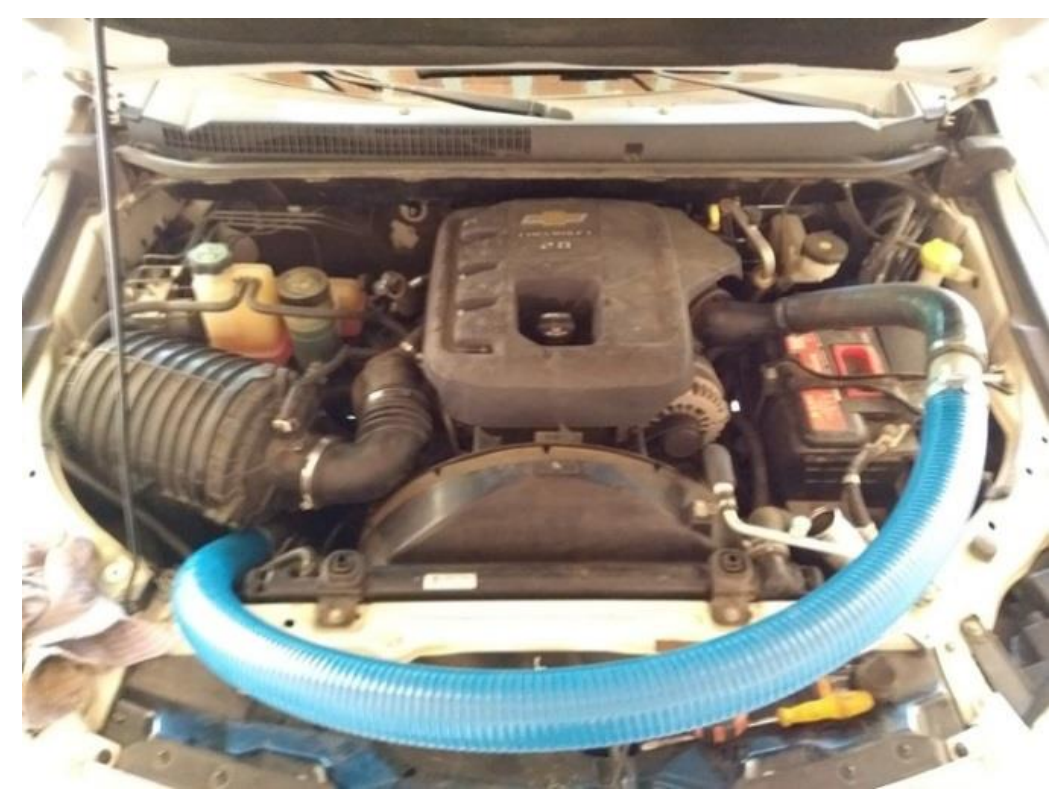

FIGURA 3. Mangueira para adaptação. Fonte: Autor (2018).

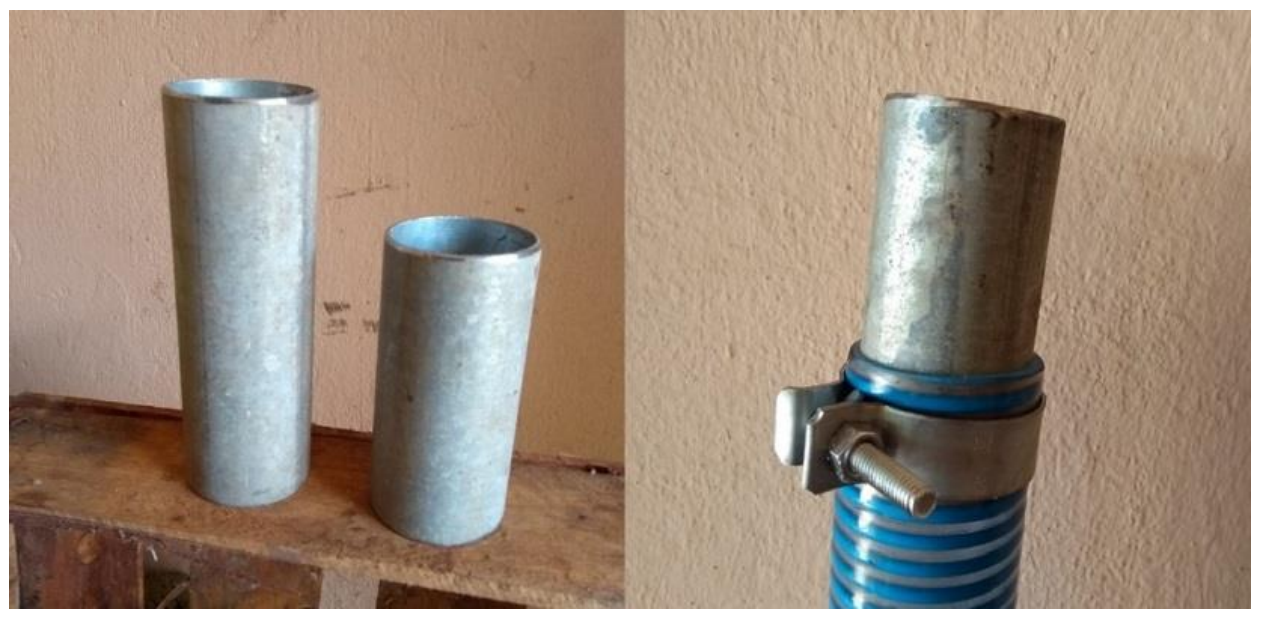

FIGURA 4. Conexões para adaptação. Fonte: Autor (2018).

Para execução destes testes no dinamômetro, foram realizadas algumas etapas e procedimentos, como:

- Colocação do automóvel de marcha à ré sobre a rampa e suas rodas de tração sobre os cilindros do dinamômetro, além da fixação do automóvel à rampa e dos cilindros através de cintas e catracas que prendem o eixo e rodas do automóvel ao equipamento;

- Colocação de mangote na descarga do automóvel para direcionar os gases do escapamento para parte externa do laboratório, conforme a Figura 5; 


\section{XIX \\ CENEMI}

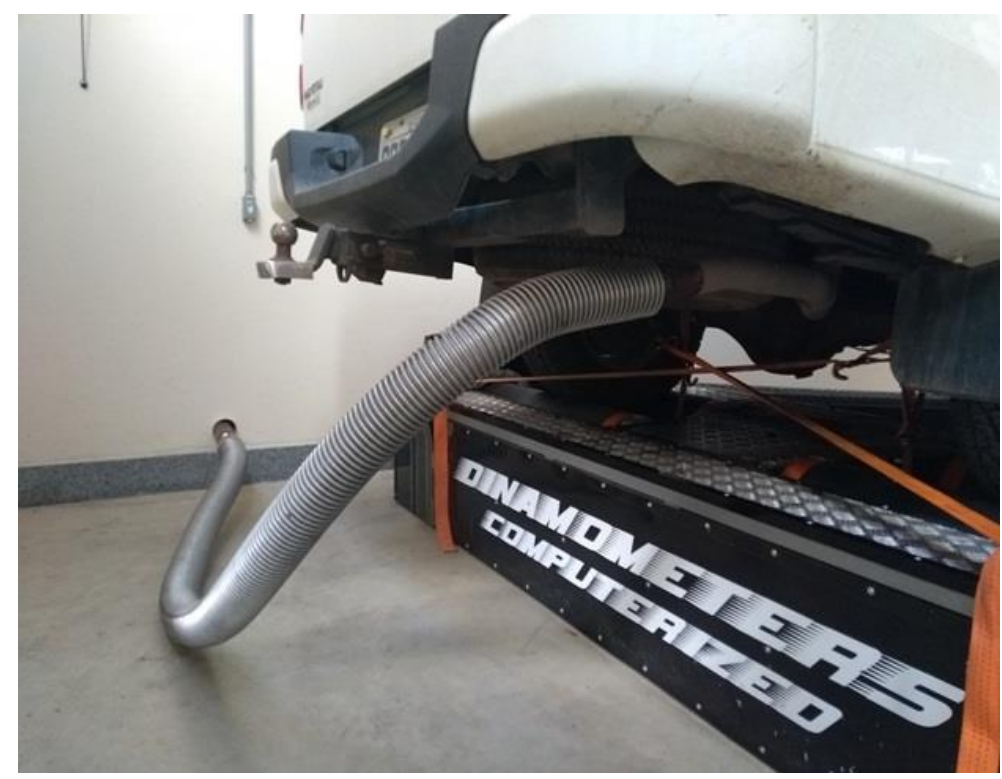

FIGURA 5. Escapamento dos gases. Fonte: Autor (2018).

- Adequação e relocação do ventilador, sendo posicionado em frente ao automóvel, simulando o fluxo do vento em um automóvel em movimento, conforme a Figura 6;

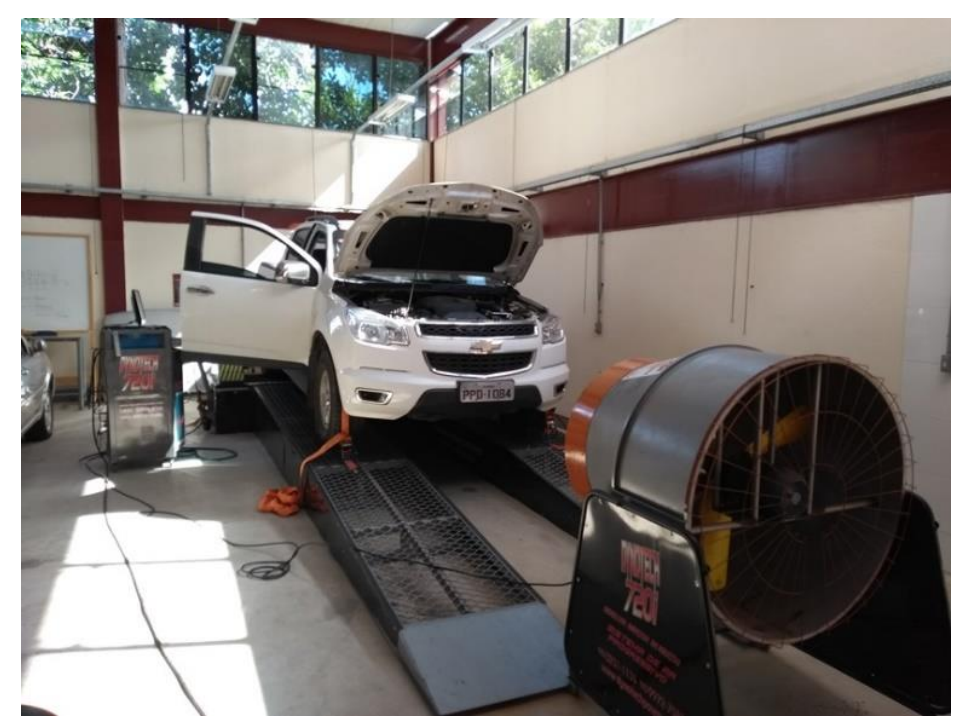

FIGURA 6. Recolocação do ventilador do dinamômetro. Fonte: Autor (2018).

- Calibração do software no dinamômetro para o automóvel testado, através do sincronismo das rotações por minuto (rpm) do motor com a velocidade do cilindro do dinamômetro;

- Acionamento e aceleração do automóvel, seguindo os padrões para teste;

- Coleta de medição de temperatura do ar admitido através de multímetro e dos dados, torque e potência do motor em suas condições originais, obtidos pelo dinamômetro; 


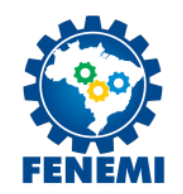

\section{$\mathbf{X I X}$ C.5NEMI}

- Desmontagem parcial da linha de admissão de ar, permitindo a adaptação, como mostram as Figuras 7 e 8, de instalação de mangueiras, tubos, conexões e adaptadores necessários para o funcionamento do motor sem o uso do intercooler;
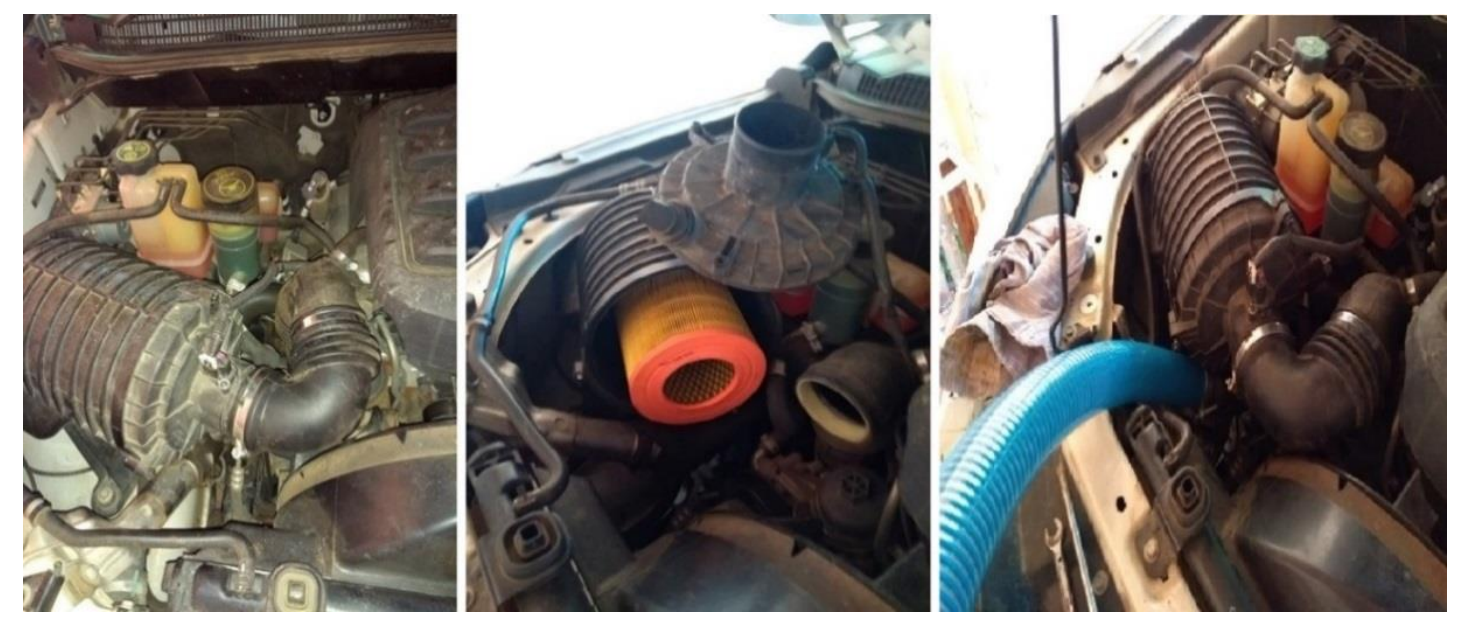

FIGURA 7. Adaptações sem o intercooler. Fonte: Autor (2018).
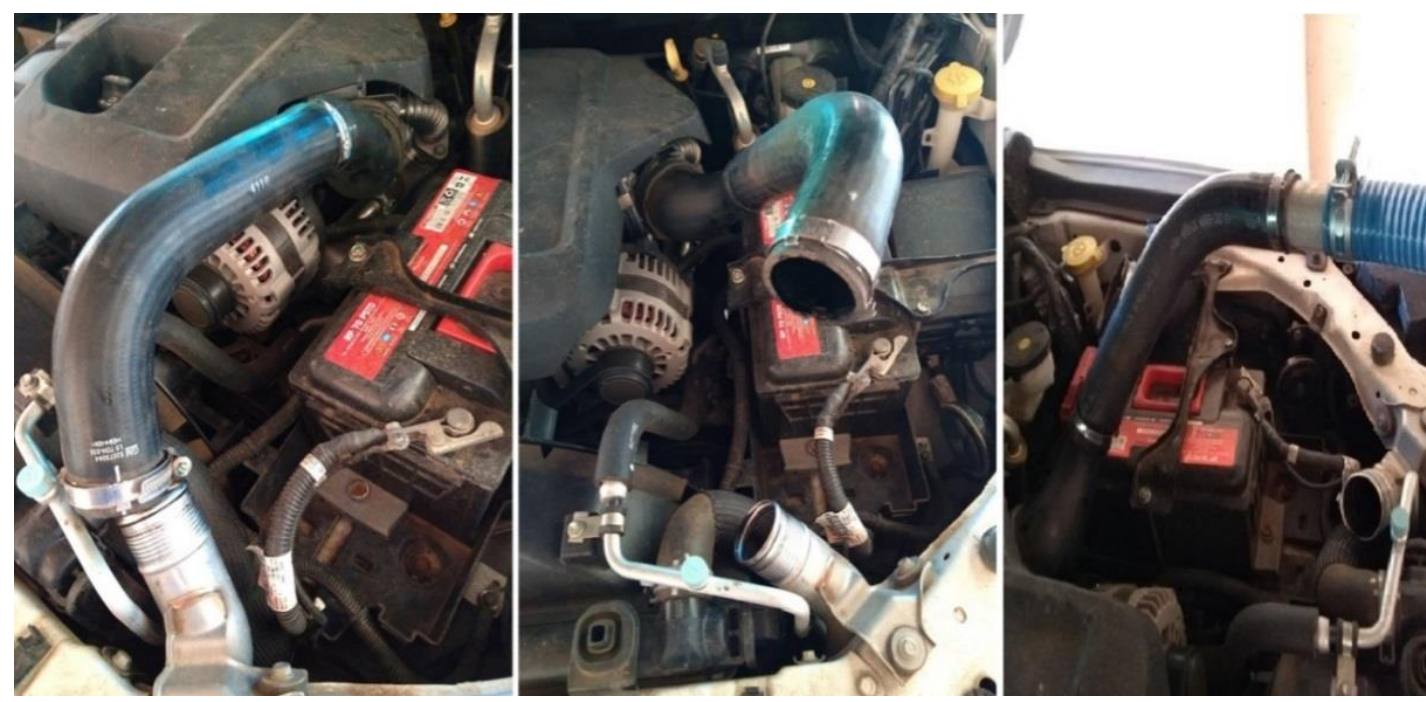

FIGURA 8. Adaptações sem o intercooler. Fonte: Autor (2018).

- Coleta de dados de temperatura do ar admitido, torque e potência do motor com as devidas alterações e sem o funcionamento do sistema de intercooler;

- Remoção das adaptações, devolvendo o automóvel às suas condições originais de funcionamento com o intercooler e retirada do automóvel do equipamento, soltando as fixações.

Para garantir que o comparativo fosse feito de maneira imparcial, foram utilizados dados e valores gerados pelos equipamentos e softwares, assim como tabelas e recursos gráficos do programa de planilhas eletrônicas do Excel. 


\section{XIX \\ CENEMI}

\section{RESULTADOS}

Foram realizados onze testes no total, onde seis foram utilizando condições originais do automóvel, com o uso do sistema de intercooler e cinco testes foram realizados sem o uso do sistema de intercooler, com as devidas adaptações. Esses testes foram realizados no mesmo dia e em sequência, evitando que possíveis fatores externos pudessem alterar os resultados.

Para efeito de validação da análise e comparação, foram desconsiderados alguns testes, devido a algumas anormalidades apresentadas na sequência dos testes do dinamômetro, como alterações no desenvolvimento de giro do motor, troca de marchas do câmbio automático, tempo de arrancada na marcha de teste e pequenos ajustes de leitura, onde poderiam trazer valores inadequados ou imprecisos.

Foram escolhidos dois testes para representar os valores com o uso do sistema de intercooler e mais dois testes para representar os valores dos testes sem o uso do sistema de intercooler.

Para melhorar o entendimento das diferenças de resultados dos testes que representa a mudança de performance do motor nas condições exigidas, foram calculadas médias desses testes e construídos gráficos para os resultados serem comparados.

Na Figura 9, é apresentado os valores médios de torque e potência com o uso do intercooler, destacando os valores de potência e torque máximos atingidos pelo automóvel durante os testes.

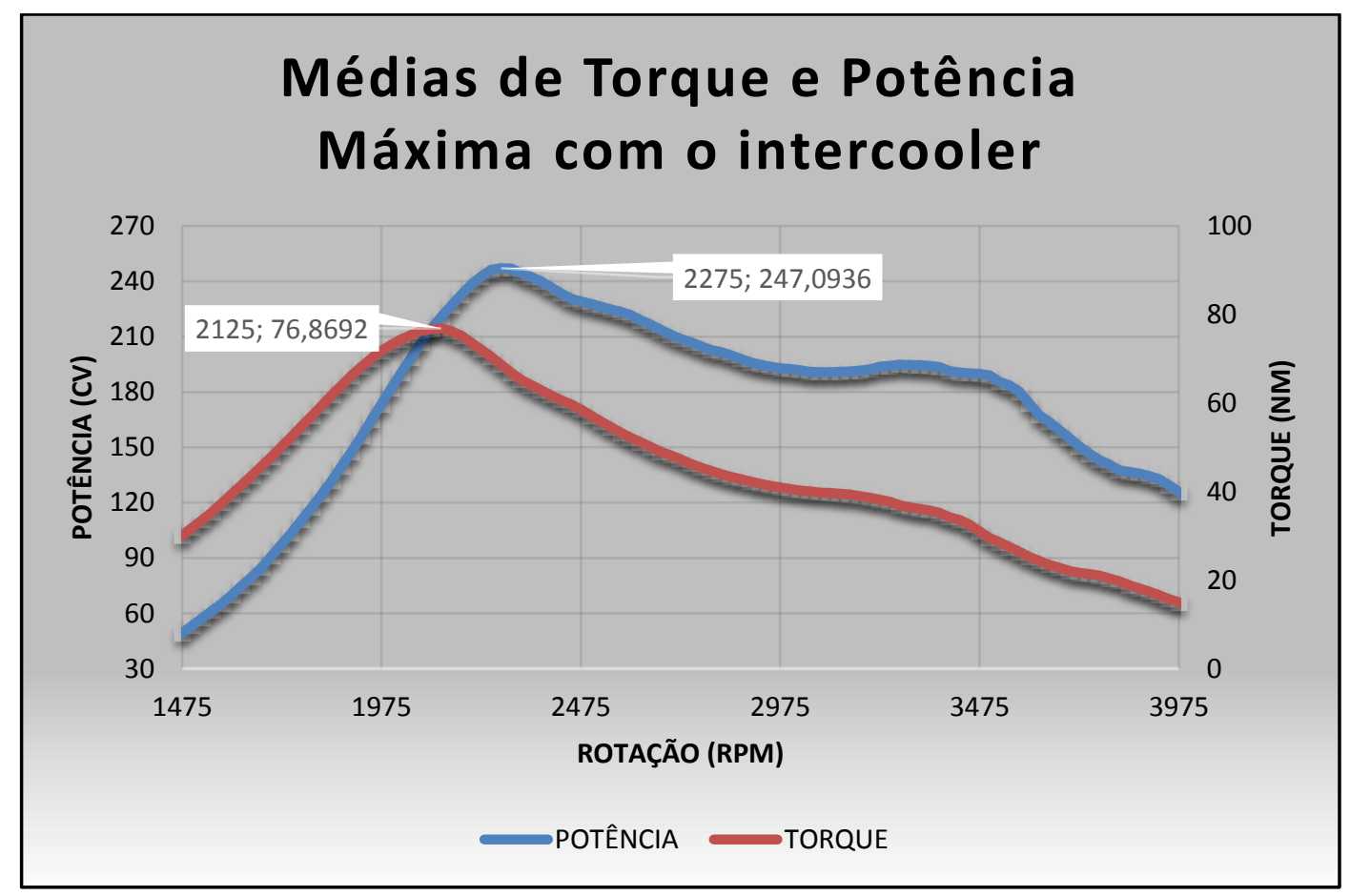

FIGURA 9. Gráfico da média de torque e potência com o uso do intercooler, Fonte: Autor (2019). 


\section{XIX \\ CENEMI}

Nestes testes, ao observar a performance e o comportamento do motor com o uso do intercooler, foi possível identificar que a temperatura máxima do ar na admissão do motor chegou a $56^{\circ} \mathrm{C}$. Tendo atingido potência máxima de 247,09 CV à $2.275 \mathrm{rpm}$ e torque de 76,87 Nm à $2.125 \mathrm{rpm}$.

Na Figura 10 são apresentados os valores médios de torque e potência durante os testes, sem o uso do intercooler, seguindo as mesmas propriedades do gráfico utilizando o intercooler, ressaltando a diferença dos dois resultados de potência máxima 167,85 CV à 2.025 rpm e torque máximos 58,11 Nm à $1850 \mathrm{rpm}$. Nestes testes, sem o uso do sistema de intercooler, a temperatura do ar na admissão do motor chegou a $112^{\circ} \mathrm{C}$.

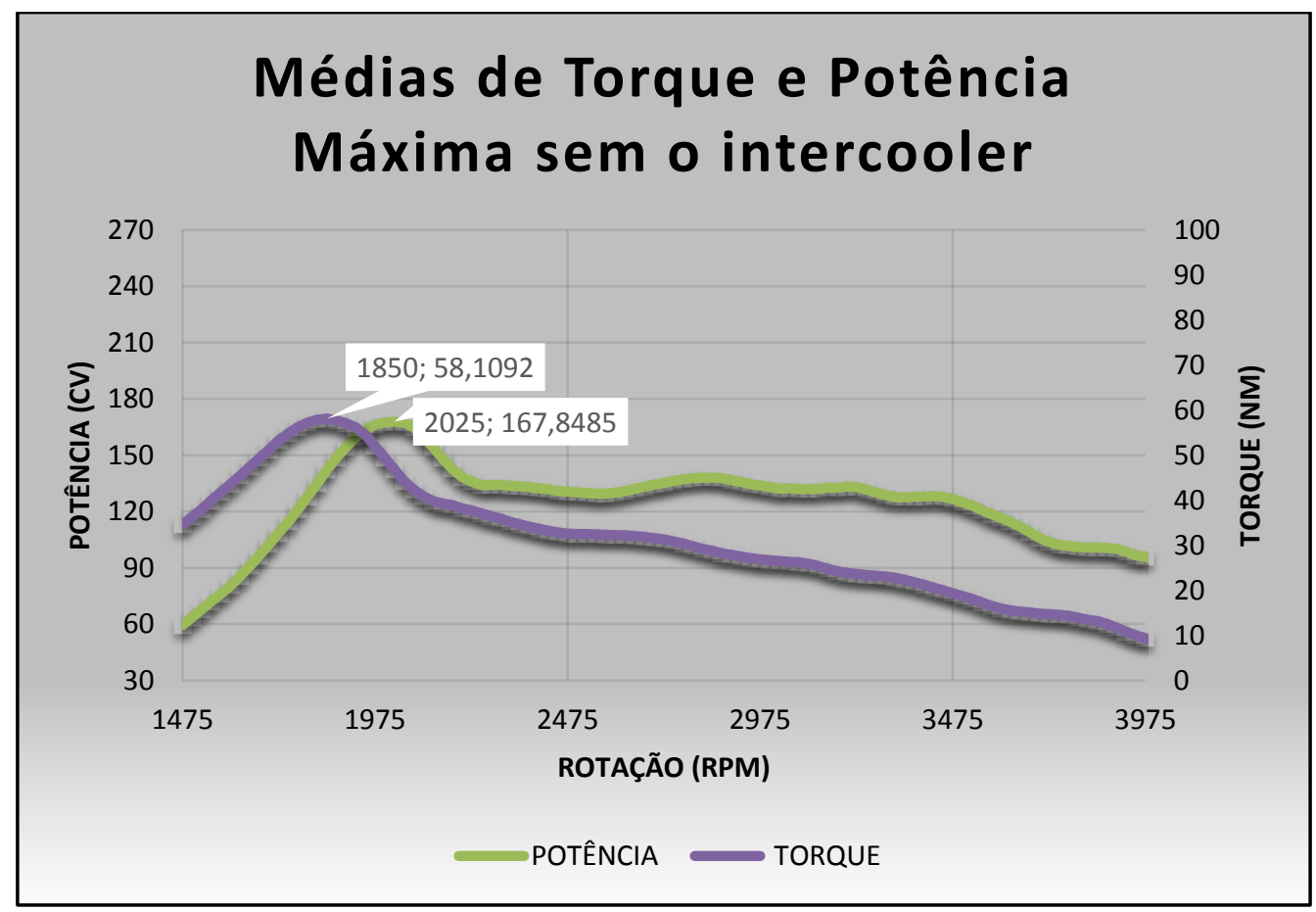

FIGURA 10. Gráfico da média de torque e potência sem o uso do intercooler. Fonte: Autor (2019).

É utilizada a Figura 11, para comparar as médias de torques encontrados através dos testes com e sem o uso do intercooler. Através de seus torques máximos, é facilmente notado que o torque com o uso do intercooler alcança valores maiores da ordem de $24,4 \%$, se comparado ao que se obtém sem o uso do intercooler, o que já era esperado, uma vez que seus pistões ganham mais forças. 


\section{XIX \\ CIONEMI}

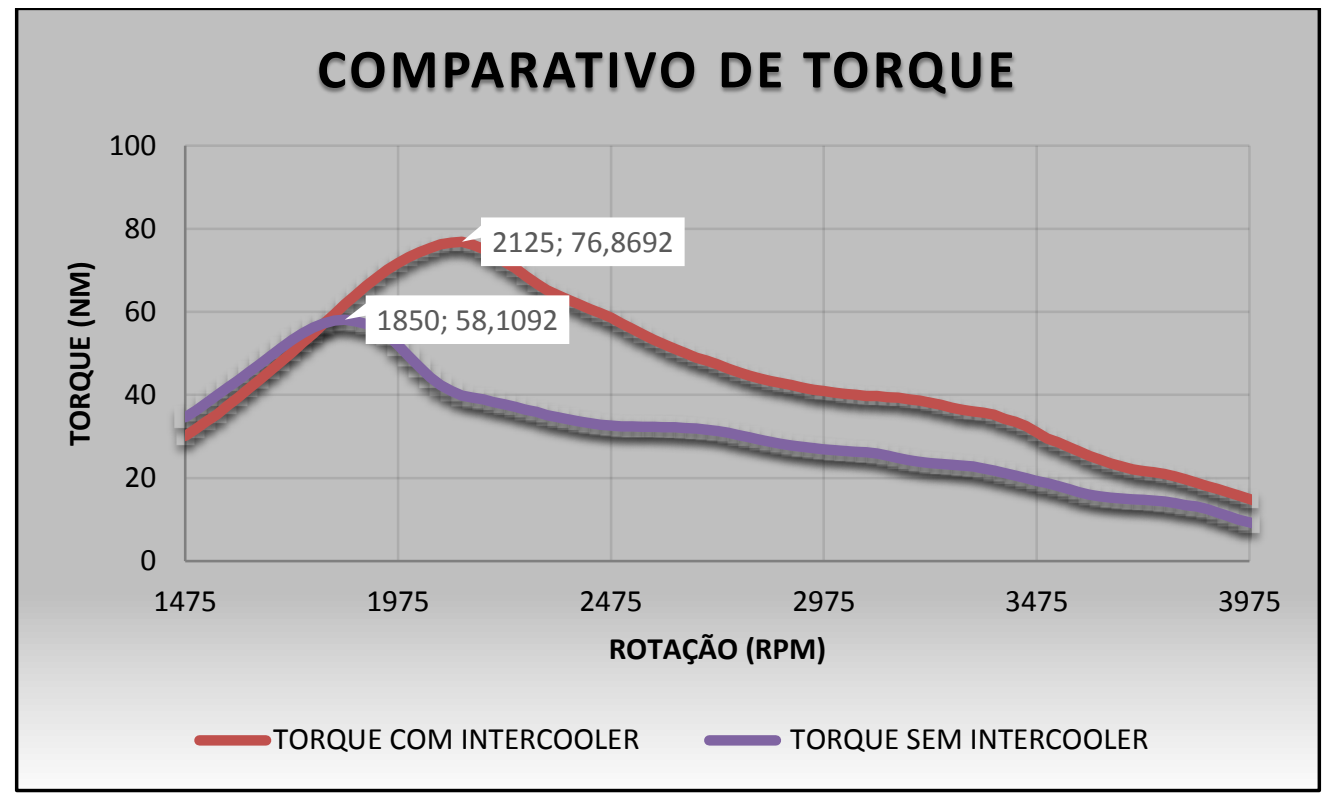

FIGURA 11. Gráfico comparativo de torque com e sem o intercooler. Fonte: Autor (2019).

Através da Figura 12, é possível comparar os valores de potência, utilizando e não utilizando o intercooler. É notável que com a presença do intercooler, esta potência alcança valores maiores do que com a sua ausência, sendo significativo de 32,1\% maior.

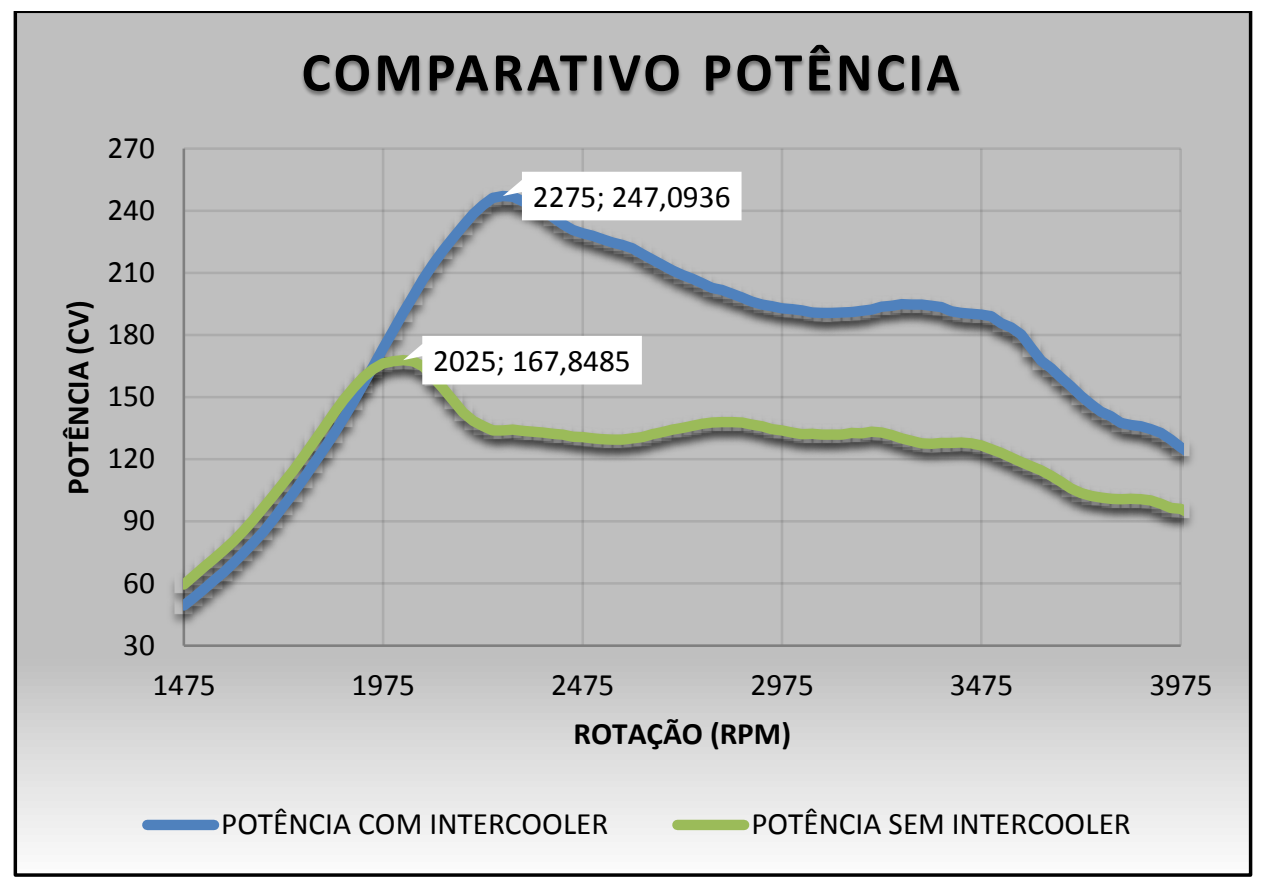

FIGURA 12. Gráfico comparativo da potência com e sem o intercooler. Fonte: Autor (2019). 


\section{$\mathrm{XIX}$ CENEMI}

Através da Tabela 2 é possível realizar a comparação de valores de torque e potência, seguidos de suas rotações. Tornando mais clara e objetiva a interpretação.

TABELA 2. Variação de torque e potência máximos.

Com o uso do intercooler
Sem o uso do intercooler

\section{Variação (\%)}

\begin{tabular}{l|c|c|c|}
\hline Torque Máximo (Nm) & 76,8692 & 58,1092 & 24,405 \\
\hline Rotação (rpm) & 2125 & 1850 & - \\
\hline Potência Máxima (cv) & 247,0936 & 167,8485 & 32,071 \\
\hline Rotação (rpm) & 2275 & 2025 & - \\
\hline
\end{tabular}

Fonte: Autor (2019).

Observando a Tabela 2, é identificado que houve uma perda de $24,40 \%$ de torque máximo sem o uso do intercooler. O mesmo acontece com a potência, respondendo a uma perda de 32,07\% quanto ao uso do veículo sem o intercooler.

Com o uso do intercooler, seu torque alcança valores maiores, identificando uma rotação equivalente a esse torque com valor de $2125 \mathrm{rpm}$, ocorrendo uma queda na curva a partir desse valor. Quando analisado o torque máximo sem o uso do intercooler, é notória uma rotação a esse torque máximo no valor de 1850 rpm, identificando uma queda na curva a partir dessa rotação.

Através da Figura 12 é possível identificar que a potência máxima produzida com o uso do intercooler é identificada a uma rotação maior, sendo está correspondente a 2275 rpm, quando comparada a ausência do intercooler, a partir de então há uma queda na curva. Quando retirado o intercooler, a potência máxima ocorre uma rotação equivalente a 2025 rpm, ocorrendo também uma queda na curva a partir deste valor.

Segundo o fabricante, sua potência máxima corresponde a 200 CV com rotação a 3600 rpm. Ao analisar o gráfico da Figura 12 em relação a essa rotação, é identificado que sem o uso do intercooler, sua potência chega a 116,5871 CV e com o uso do intercooler, essa potência tem o valor de 173,695 CV, se aproximando da potência fornecida pelo fabricante através da Tabela 1, porém quando comparados com a potência máxima obtida nos testes com a fornecida pelo fabricante, é identificado através da Tabela 2 que com o uso do intercooler sua potência ultrapassa a potência máxima informada pelo fabricante, diferente da potência obtida através dos testes sem o intercooler, que sua potência máxima é menor que a potência máxima informada pelo fabricante.

Na Figura 11 é analisado o torque do veículo. O fabricante identifica um torque máximo a 51 kgfm a 2000 rpm. Quando analisados os torques obtidos pelos testes nessa mesma rotação, é 


\section{$\mathbf{X I X}$ \\ CIONEMI}

identificado um torque com o uso do intercooler a 71,7902 Nm e sem o uso do intercooler a 49,4214 $\mathrm{Nm}$, sendo o torque sem intercooler um pouco abaixo, enquanto utilizando o intercooler fica acima do torque fornecido pelo fabricante. Através da Figura 11, é apontado um toque máximo sem o uso do intercooler com valor de 58,1092 kgfm a com o uso do intercooler 76,8692 kgfm, ambos torques atingindo valores maiores que o fornecido pelo fabricante.

\section{CONCLUSÕES}

No acompanhamento dos testes e observação do funcionamento e comportamento do motor, foi observado que o intercooler foi capaz de resfriar o ar e diminuir a temperatura de entrada na admissão do motor, onde a temperatura máxima chegou a $56^{\circ} \mathrm{C}$ com o uso do intercooler e sem o uso do intercooler a temperatura encontrada foi de $112^{\circ} \mathrm{C}$.

Consequentemente houve alteração na densidade do ar, onde o ar em temperaturas mais altas, é menos denso, ou seja, essa redução de temperatura, esse resfriamento do ar que é feito pelo intercooler, diminuindo a temperatura do ar de $112^{\circ} \mathrm{C}$ que sai do turbocompressor para uma temperatura de $56^{\circ} \mathrm{C}$ na admissão do motor, o torna bem mais denso, com mais oxigênio, com mais massa de ar para um mesmo volume do cilindro; com isso, produz uma melhor queima de combustível, gerando mais força no motor. Esse aumento da massa de ar produz uma melhora na combustão, que é o estágio do ciclo responsável pela geração de força motriz no eixo do motor, o qual é a fonte fornecedora de torque e potência.

Essa diferença de temperatura, ou melhor, esta diminuição da temperatura do ar, com o uso do intercooler, foi o grande responsável pelos maiores valores de torque e potência encontrados nos testes realizados, onde os valores de tabelas e gráficos apresentados, demonstram isso facilmente; validando e comprovando de que o uso do intercooler é grande responsável pelo ganho de torque e potência de um motor turbo alimentado, e que o uso do intercooler está diretamente relacionada à performance do motor.

Para este motor, a diferença do resultado e o ganho de torque e potência são extremamente significativos, mas levadas às devidas proporções, há de se imaginar o quão significativo seria em um motor de grande porte, de grande força exigida, o uso do intercooler para melhorar seu rendimento e sua performance. 


\section{XIX \\ CIONEMI}

\section{REFERÊNCIAS}

BRUNETTI, Franco. Motores de Combustão Interna. São Paulo: Edgard Blucher Ltda, 2012.

HEYWOOD, J. B. Internal Combustion Engine Fundamentals. McGraw-Hill Book CO.; New York, 1988.

MARAN, Melsi. Diagnósticos e regulagens de motores de combustão interna: Área automotiva. São Paulo: SENAI-SP Editora, 2013.

MARTINS, Jorge. Motores de combustão interna. 4.ed. São Paulo: Publindústria, 2013.

MOREIRA, F. S. Diagnóstico de um Motor Diesel por Análise dos Gases de Descarga e Simulação de seu Ciclo de Funcionamento, Dissertação de M.Sc. Rio de Janeiro: COPPE/UFRJ, 2000.

TILLMANN, Carlos Antonio da Costa. Motores de Combustão Interna e seus Sistemas. Pelotas: IFES, 2013.

\section{AGRADECIMENTOS}

Agradecemos a Universidade Candido Mendes por disponibilizar o laboratório para realização dos testes e ao Prof. André Luiz Vicente de Carvalho pela disponibilidade e colaboração. 